\title{
Rethinking "Ecological Migration" and the Value of Cultural Continuity: A Response to Wang, Song, and Hu
}

\author{
J. Marc Foggin
}

Received: 9 October 2010/Accepted: 13 October 2010/Published online: 16 November 2010

This comment was not peer reviewed.

Comment to: Wang Z.M., K.S. Song, and L.J. Hu. 2010. China's largest scale ecological migration in the three-river headwater region. AMBIO 39 (5-6):443-446.

The vast grasslands of the Tibetan Plateau constitute a critically important environment providing many key ecological services such as carbon storage, soil erosion control, and water regulation. The Three-River Headwater Region of the Plateau-also known as the Sanjiangyuan region; encompassing the source areas of the Yangtze, Yellow and Mekong rivers with a total area of $363,000 \mathrm{~km}^{2}$, larger than Germany-is particularly significant for China and Southeast Asia. Wang et al. (2010) are correct to encourage its conservation. However, this land also is home to around 300,000 Tibetan pastoralists who have adapted their way of life over generations and learned to live successfully in the harsh, often unpredictable environment. Now, several conservation-oriented government policies such as Ecological Migration (EM) are threatening not only local pastoralists' livelihood and community structure, but also regional stability as quotadriven resettlements are married with high levels of unemployment and loss of hope (Foggin 2008).

EM is all too often accepted with little critique in China. For example, Wang et al. (2010) fail even to mention that EM could, just possibly, best be abandoned. Despite some of the clear social and environmental challenges that already have begun to emerge, as indicated by the authors

The online version of the article commented upon can be found at doi:10.1007/s13280-010-0054-z themselves, they still positively encourage its continuation and expansion. Such a position is astonishing, since the EM policy remains an untested social experiment at an enormous scale-with potential devastating long-term (generational) social, cultural, and possibly environmental consequences; some of them irreversible.

The primary environmental rationale behind EM is largely inadequate, in as much as sufficient scientific evidence for both the scale and the underlying causes of grassland degradation are still lacking (Harris 2010); and even where there is observed degradation, this still does not warrant or necessarily point toward EM as the only possible solution. More importantly, several alternate approaches to conservation already exist, including certain traditional resource management practices and other tried-and-tested forms of local governance such as co-management schemes (Banks et al. 2003; Reed 2008). In addition, due to the expected impacts of relocation and resettlement on such a large number of people (some media suggest plans for over half a million people in the province to be "settled" permanently), development and conservation agendas should be more carefully integrated in the future. A relatively new approach to public policy research and analysis is, therefore, recommended-horizontal policy coordination (not only a vertical, insular analysis within single fields or government bureaus) - so that the complex issues of conservation and sustainable development can be considered more effectively in an increasingly globalized world.

Now before embarking on such an irreversible, largescale, and untested social experiment... Would it not be wise to learn from other similar experiences elsewhere in the world?

In Canada, for instance, it has become apparent that working with local communities as key partners, toward common goals, is more effective than simply legislating for 
desired behaviors. In some cases, communities live inside protected areas and work closely with national park authorities. In other cases, community co-management approaches have been adopted wherein two or more parties share equally in the varied responsibilities of management for conservation and development. In certain situations, new public-private partnerships are being developed in an effort to better protect the environment at the same time as providing employment, developing the economy and enhancing visitors' experiences in national parks. In several First Nations, the most effective form of partnership has been a co-management agreement between local people and national parks, with varying levels of shared responsibilities ranging from simple participation to more autonomous forms of governance and leadership. Such approaches have helped achieve the connected goals of sustainability and conservation. It is thus clear from the Canadian experience that governance matters.

Working with people as genuine partners-allowing them the possibility to continue their way of life as pastoralists, but with scope to adapt and modify their livelihoods; not seeking to preserve any person or culture as a static museum piece-this too is essential to help achieve fair and equitable development. In Canada, the public health situation of many First Nations' communities is but one flagrant example, together with the legacy of the residential school system, of the devastation that can be caused by failed partnerships. It is also what such programs removed from some communities-a sense of identity, and hope for the future - that now is demonstrated in the public health literature to be critically important protective features for many aboriginal people.

Indeed, in a very significant way, cultural continuityas measured by a suite of factors including availability of culturally relevant and accessible health programs, education programs, local governance, and community centersis a very important feature that government, with communities, can strive to develop well. Each of the above variables can affect health independently, yet synergistically they are even more powerful predictors of health and well-being (Chandler and Lalonde 1998). Success in this area alone will help lead to happier and healthier communities, while failure will pave the way to falling health indices, increased societal confusion, and ultimately to greater financial burden on the entire nation. The outcome of such present decisions-that is, to promote or not to promote cultural continuity-will be long-lasting, possibly spanning many generations.
For Tibetan pastoralists in the Sanjiangyuan region where the EM policy is presently moving forward most rapidly, the implications are clear. Specifically, the scope or extent to which the policy is implemented should be constrained; new approaches to conservation that allow greater stakeholder participation should be further researched, trialed, and adopted where appropriate; and more accessible forms of service provision should be developed, both for herders still living in grassland areas and (former) herders now living in new towns. With social concerns over EM already raised, and proven alternative means such as co-management available to reach desired conservation goals, EM should no longer be promoted regardless of consequences. Rather, some of the important lessons learned in Canada and elsewhere through systematic, scientific analyses should be taken seriously-and more sustainable, equitable, and economic measures for long-lasting development and environmental conservation introduced to the grasslands of the Tibetan Plateau.

\section{REFERENCES}

Banks, T., C. Richard, and Z.L. Yan. 2003. Community-based grassland management in Western China: Rationale, pilot project experience, and policy implications. Mountain Research and Development 23(2): 132-140.

Chandler, M.J., and C.E. Lalonde. 1998. Cultural continuity as a hedge against suicide in Canada's First Nations. Transcultural Psychiatry 35(2): 193-211.

Foggin, J.M. 2008. Depopulating the Tibetan Grasslands: National policies and perspectives for the future of Tibetan Herders in Qinghai Province, China. Mountain Research and Development 28(1): $26-31$.

Harris, R. 2010. Rangeland degradation on the Qinghai-Tibetan plateau: A review of the evidence of its magnitude and causes. Journal of Arid Environments 74: 1-12.

Reed, M.S. 2008. Stakeholder participation for environmental management: A literature review. Biological Conservation 141: $2417-2431$.

Wang, Z.M., K.S. Song, and L.J. Hu. 2010. China's largest scale ecological migration in the three-river headwater region. AMBIO: A Journal of the Human Environment 39(5-6): $443-446$.

J. Marc Foggin ( $\square)$

Address: Plateau Perspectives Canada, 200 Walnut Ave, St Lambert, QC J4P 2T1, Canada.

Address: School of Geography and Life Sciences, Qinghai Normal University, 38 Wusi Dajie, 810008, Xining, Qinghai, People's Republic of China.

e-mail: foggin@plateauperspectives.org 